Lingle, Wilma

[42]

\section{Investigation of gene expression correlating with centrosome amplification in development and progression of breast cancer}

Wilma Lingle ${ }^{1}$, Stephen Iturria ${ }^{1}$, Patrick Roche ${ }^{1}$, Fergus Couch ${ }^{1}$, James Ingle ${ }^{1}$, Youzhen Wang ${ }^{2}$, Kathleen Steinmann², Yong Yao $\mathrm{Xu}^{2}$, Maureen Mertens², Yueying $\mathrm{Cao}^{2} \&$ James Lillie ${ }^{2}$

${ }^{1}$ Mayo Clinic Cancer Center, Rochester, Minnesota, USA

${ }^{2}$ Millennium Predictive Medicine, Cambridge, Massachusetts, USA

We have developed differential gene expression data sets from breast tumors through analysis of 8 in situ and 32 invasive breast tumors relative to 6 samples of normal breast tissue using arrays containing 40,000 DNA elements. Depending on the cutoff values used, $10-20 \%$ of these sequences are differentially expressed in breast tumors compared with normal breast tissues. We have taken the new approach of using centrosome amplification, a tumor-associated phenomenon, to rank tumors so that genes associated with this phenomenon can be identified for further study. Centrosome amplification has recently been identified as a feature common to many human tumors, including those of the breast. Proper structure and function of duplicated mitotic centrosomes are required for fidelity in chromosome segregation and preservation of diploidy. We have shown that in breast tumors centrosome amplification correlates with aneuploidy. In the present work, the degree of centrosome amplification was quantified by immunofluorescence microscopy. We ranked tumors by their degree of centrosome amplification, and genes whose expression strongly correlated with this ranking were identified. We are testing the biological significance of genes identified in this manner in a cell culture assay system designed to measure the effects of their overexpression on the structural and functional integrity of the centrosome and mitotic spindle apparatus. Given the obligate requirement for proper structure and function of mitotic and interphase centrosomes in regulation of mitosis and cell polarity, the identified subset of genes associated with centrosome amplification is probably involved in the cell cycle and the development of aneuploidy, both of which are key factors in cancer development and progression.

Linja, Marika

\section{Quantification of androgen receptor gene expression in prostate carcinomas with a real-time RT-PCR assay}

Marika Linja, Kimmo Savinainen, Outi Saramäki, Teuvo Tammela \& Tapio Visakorpi

\section{Laboratory of Cancer Genetics, University of Tampere and TAUH, Finland}

Androgen deprivation is the standard treatment for advanced prostate cancer. It is initially highly effective, causing cessation of growth and induction of apoptotic death of androgen-dependent tumor cells. It has been shown that both primary and hormone-refractory prostate cancers express the androgen receptor (AR) protein. However, eventually a recurrent, hormone-refractory tumor emerges. In addition, amplification of the AR gene has been found in 30\% of recurrent tumors and AR overexpression due to gene amplification has been suggested by nonquantitative in situ hybridization of messenger RNA. This study measured AR gene expression in both hormone-naive and refractory prostate tumors. We have set up a method for the quantitation of AR and prostate-specific antigen expression using sequence detection by means of the Light Cycler real-time polymerase chain reaction with reverse transcription (RT-PCR). We normalized measurements by measuring the amount of mRNA from testosterone-binding protein in each sample. We analyzed 33 untreated and 13 hormone-refractory tumor samples as well as 8 benign prostatic hyperplasias using RT-PCR. On average, hormone-refractory prostate tumors expressed sixfold higher levels of AR than untreated tumors or benign prostatic hyperplasias $(P<0.001$; Kruskall-Wallis test). Benign prostatic hyperplasias and untreated tumors expressed more than twice the amount of prostate-specific antigen than hormone-refractory tumors $(P<0.05)$. The analysis of AR copy number by fluoresence in situ hybridization is continuing. The results clearly demonstrate that hormone-refractory tumors highly express the AR gene, further supporting the notion that AR plays a central role in the failure of hormonal treatment.

Liu, Hsiao-Ching

$[44]$

\section{Growth hormone interacts with the Marek's disease virus SORF2 protein and is associated $w$ ith disease resistance in chicken} Hsiao-Ching Liư ${ }^{1}$, Hsing-Jien Kung ${ }^{2}$, Janet Fulton
Morgan

${ }^{1}$ Avian Disease and Oncology Laboratory, U.S. Department of Agriculture, Agricultural Research Service, East Lansing, Michigan, USA

${ }^{2}$ University of California Davis Cancer Center, Sacramento, California, USA

${ }^{3} \mathrm{H} y$-Line International, Dallas Center, Iowa, USA

${ }^{4}$ Department of Animal and Food Science, University of Delaware, Newark, Delaware, USA

Marek's disease (MD) is a T-cell lymphoma of chickens induced by a herpesvirus, the Marek's disease virus. Since MD is a significant economic problem to the poultry industry, there is great interest in enhancing genetic resistance, which is controlled by multiple genes. MD is also a biomedical model, as vaccines prevent disease formation but do not prevent viral replication. The influence of the major histocompatibility complex has been clearly demonstrated, and several quantitative trait loci have been mapped; however, no single gene influencing MD resistance has been identified. Transcription of SORF2 is perturbed in the Marek's disease virus recombinant clone RM1 owing to a solo insertion of the reticuloendotheliosis virus long terminal repeat, which may explain the loss of oncogenicity for this strain. Hypothesizing that SORF2-interacting host proteins are involved in MD resistance, we screened a chicken splenic complementary DNA library by the yeast two-hybrid assay using SORF2 as bait. The chicken growth hormone structural peptide was identified and the specific interaction verified by co-immunoprecipitation. Immunohistochemical staining and indirect immunofluorescence assay indicated that growth hormone and SORF2 are expressed and can be colocalized in cells infected with the Marek's disease virus and MD tumors. Furthermore, depending on the major histocompatibility complex genotype, polymorphism in the growth hormone gene (GH1) is associated with the number of tumors in a commercial chicken resource population. We conclude that $\mathrm{GH} l$ is likely to be an MD resistance gene. Furthermore, a systematic search of virus-host protein interactions should complement our quantitative trait loci scans and DNA microarrays to identify other resistance genes to MD. 